\title{
Perfil do paciente atendido pela fonoaudiologia na unidade de urgência e emergência de um hospital universitário
}

\author{
The profile of a patient receiving speech-language therapy \\ assistance at a school hospital emergency unit
}

Karla Cristina Freitas Faria', Ana Cláudia Neres Pessoa' ${ }^{1}$ Lúcia Inês Araújo ${ }^{1}$, Maria Luíza de Faria Paiva ${ }^{1}$

\begin{abstract}
RESUMO
Objetivo: Caracterizar a população atendida pelos residentes de Fonoaudiologia no pronto-socorro de um hospital universitário. Métodos: A amostra foi composta por 150 sujeitos de ambos os gêneros, na faixa etária de 20 anos a 59 anos e acima de 60 anos, internados no período de março de 2010 a março de 2012. Resultados: Houve predominância de pacientes do gênero masculino, idosos, com ensino fundamental incompleto e com baixa renda, procedentes, em sua maioria, da capital, Goiânia, e região metropolitana. No diagnóstico médico, predominaram as doenças do aparelho circulatório, doenças do aparelho digestivo e doenças do sistema nervoso. Os diagnósticos fonoaudiológicos encontrados foram: deglutição normal/funcional, disfagia orofaríngea neurogênica e disfagia esofágica. Conclusão: Faixa etária associada a doenças de base prevalentes pode levar a distúrbios da deglutição, podendo agravar o quadro e prolongar o período de permanência do sujeito na instituição, sendo de extrema importância a presença do fonoaudiólogo nos serviços de urgência e emergência, para avaliar, diagnosticar e tratar os distúrbios deglutitórios.
\end{abstract}

Descritores: Disfagia; Adulto; Idoso; Tratamento de emergência; Fonoaudiologia

\begin{abstract}
Purpose: This paper sought to evaluate patients receiving assistance from resident speech-language therapists at a school hospital emergency room. Methods: Both adult and elderly patients were included in this study. In total, 150 individuals of both genders, including adults between the ages of 20-59 years and elderly individuals over 60 years of age, who were hospitalized from March 2010 to March 2012 were studied. Results: The patients were predominantly male and elderly, with incomplete elementary school education and a low income, and originated from the capital city and metropolitan region. The medical diagnoses predominantly revealed circulatory diseases, digestive disorders, and nervous system disorders. The speech-language therapy diagnoses showed normal swallowing function, neurogenic oropharyngeal dysphagia, and esophageal dysphagia. Conclusion: The prevalence of age-related systemic disorders may lead to swallowing disorders, which could trigger complications such as longer hospital stays. Therefore, the presence of a speech-language therapist in an emergency room is important to assess, diagnose, and treat swallowing disorders.
\end{abstract}

Keywords: Dysphagia; Adult; Aged; Emergency treatment; Speech, Language and Hearing Siences

Trabalho realizado no Programa de Residência Multiprofissional em Saúde, área de concentração Urgência e Emergência, Hospital das Clínicas, Universidade Federal de Goiás - UFG - Goiânia (GO), Brasil.

(1) Programa de Residência Multiprofissional em Saúde, área de concentração Urgência e Emergência, Hospital das Clínicas, Universidade Federal de Goiás UFG - Goiânia (GO), Brasil.

Conflito de interesses: Não

Contribuição dos autores: $F K C F$ pesquisador principal, elaboração da pesquisa, elaboração do cronograma, levantamento da literatura, coleta e análise dos dados, redação e correção do artigo, submissão e trâmites do artigo; $P A C N$ levantamento da literatura, coleta e análise dos dados; $A L I$ orientadora, elaboração da pesquisa, correção da redação do artigo; $P M L F$ coorientadora, correção da redação do artigo.

Endereço para correspondência: Karla Cristina Freitas Faria. R. Anita Garibalde, 459, Centro, Nerópolis, Goiás (GO), Brasil, CEP: 75460-000.

Email: fga-karlacff@ hotmail.com

Recebido em: 1/3/2013; Aceito em: 11/9/2013 


\section{INTRODUÇÃO}

Um dos mais atuais e sucintos conceitos para a Fonoaudiologia, entende-a como um segmento da área da saúde que compreende ações individuais e coletivas, que visam a promoção, a proteção e a recuperação da saúde, nos aspectos da linguagem, voz, audição e motricidade orofacial ${ }^{(1)}$.

À medida que seu campo de atuação é ampliado surgem novas especialidades na área e uma das mais recentes é a Disfagia. Essa especialidade é regulamentada pela Resolução $n^{\circ} 356$ de 6/12/2008, que "dispõe sobre a competência técnica e legal do fonoaudiólogo para atuar nas disfagias orofaríngeas"(2). Denomina-se disfagia o distúrbio de deglutição que acomete todo o trato digestivo - que vai da boca até o estômago -, ou parte dele ${ }^{(3)}$.

Estando a deglutição dividida em três fases, oral, faríngea e esofágica, as alterações que ocorrem nas duas primeiras fases acarretam a disfagia orofaríngea e já as alterações na última fase levam a disfagia esofágica. Do ponto de vista etiológico, as disfagias podem ser divididas em: neurogênicas, quando causadas por alguma afecção no sistema nervoso central e/ou periférico e mecânicas, quando desencadeadas por alterações anatômicas de qualquer origem ${ }^{(4)}$. Quanto à gravidade, podem ser classificadas em leve, moderada e severa ${ }^{(5)}$.

A atuação fonoaudiológica com pacientes disfágicos acontece principalmente, no ambiente hospitalar e tem conquistado cada vez mais espaço, uma vez que doenças crônicas não transmissíveis (DCNT), tais como o câncer, as doenças respiratórias, o diabetes e as doenças do aparelho circulatório, apresentam aumento significativo e, consequentemente, ocasionam alterações na deglutição. O Ministério da Saúde (MS) aponta que as DCNT são responsáveis por 45,9\% da carga global de doença no mundo, segundo a Organização Mundial de Saúde (OMS). No Brasil, essas doenças representam $75 \%$ dos gastos com atenção à saúde no Sistema Único de Saúde (SUS) ${ }^{(6)}$.

As contribuições da Fonoaudiologia, nesse contexto, são avaliação, diagnóstico e intervenção junto aos pacientes disfágicos, a fim de evitar e/ou minimizar possíveis complicações clínicas $^{(7-9)}$.

A disfagia está associada ao aumento do risco de complicações pulmonares por aspiração de saliva e/ou alimento, desnutrição, desidratação, internação prolongada e morte. Além disso, envolve fatores psicossociais e econômico-financeiros ${ }^{(10-14)}$.

No entanto, o fonoaudiólogo não é o único profissional que atua na área da disfagia. A eficácia da intervenção terapêutica dependerá da contribuição de outros profissionais, como médicos de diversas especialidades, nutricionistas, psicólogos e demais especialistas ${ }^{(7,15,16)}$. Atualmente, essa forma de atuação em equipe ganha força em todos os tipos de serviços de saúde, inclusive nas unidades de urgência e emergência, a partir da estruturação dos programas de residência multiprofissional. A presença de vários profissionais de áreas diferentes, além de otimizar o atendimento ao paciente, mostra-se benéfica quando se trata de custo/benefício ${ }^{(17,18)}$.

O fonoaudiólogo é o profissional capacitado a avaliar precocemente a deglutição e a estabelecer a via mais segura de alimentação. Sendo a unidade de urgência e emergência o setor que presta o primeiro atendimento ao paciente, é interessante que cada pronto-socorro (PS) conte com a atuação desse profissional, uma vez que existe grande demanda de pacientes potencialmente disfágicos ${ }^{(19,20)}$.

Este estudo teve como objetivo caracterizar a população atendida pelos residentes de Fonoaudiologia no serviço de urgência e emergência do Hospital das Clínicas da Universidade Federal de Goiás (HC/UFG), no período de março de 2010 a março de 2012.

\section{MÉTODOS}

Trata-se de estudo de corte transversal retrospectivo e, conforme parecer 078/2011, foi avaliado e aprovado pelo Comitê de Ética em Pesquisa do Hospital das Clínicas da Universidade Federal de Goiás (UFG). O presente estudo está inserido no Projeto de Pesquisa "Caracterização dos Atendimentos Realizados no HC/UFG”, em que foi dispensada a assinatura do Termo de Consentimento Livre e Esclarecido por não haver contato direto com os pacientes, por não identificá-los e por não causar-lhes nenhum dano moral ou material.

Foram feitas revisões bibliográficas relacionadas aos temas pertinentes, utilizando-se ferramentas de busca, como a Biblioteca Virtual em Saúde (BVS) e a Scientific Electronic Library Online (SciELO), consultas ao livro de registros de atendimentos do serviço de Fonoaudiologia do HC/UFG e, por último, busca em prontuários dos pacientes, por meio de formulário previamente elaborado pelos pesquisadores (Anexo 1).

O serviço de Fonoaudiologia desse hospital realizava atendimentos no leito somente quando recebia solicitação da equipe médica e/ou multiprofissional. Após a implementação da Residência Multiprofissional em Saúde, juntamente com a participação de profissionais da Fonoaudiologia, o serviço iniciou a captação de pacientes no leito por meio de triagem para o risco de disfagia, realizando a avaliação da deglutição após liberação médica.

Foram incluídos no estudo os pacientes adultos e idosos de ambos os gêneros (adultos de 20 a 59 anos e idosos acima de 60 anos), conforme a classificação utilizada pelo Ministério da Saúde (MS) nos calendários de vacinação ${ }^{(21)}$. Esses pacientes encontravam-se internados no pronto-socorro (PS) do HC/UFG e foram atendidos pelos residentes de Fonoaudiologia, no período de março de 2010 a março de 2012.

Foram excluídos pacientes cujos prontuários não foram localizados, ou que estavam incompletos quanto às variáveis adotadas pelo estudo. Sendo assim, o total da amostra que, inicialmente, era de 307 participantes, passou para 150.

Dados gerais como nome, sexo, idade, procedência, 
escolaridade, renda, situação funcional e diagnóstico médico foram coletados e, para a divisão das patologias, foi utilizado o Código Internacional de Doenças (CID10) ${ }^{(22)}$. Os dados específicos foram divididos em forma de acesso ao paciente, diagnóstico fonoaudiológico e número de avaliações e terapias realizadas. A coleta iniciou-se no dia 06/08/2012 e foi concluída no dia 11/10/2012.

Dos 150 participantes da pesquisa, 93 (62\%) eram do gênero masculino e 57 (38\%), do gênero feminino. Desses sujeitos, $80(53,3 \%)$ procediam de Goiânia ou região metropolitana, 66 (44\%) do interior e apenas 4 (2,7\%), de outros estados.

Em relação à faixa etária, predominou o número de pacientes idosos, que foi de $85(56,7 \%)$. Quanto aos pacientes adultos, de 20 a 59 anos, o número foi de $65(43,3 \%)$ atendidos.

\section{RESULTADOS}

Os dados socioeconômicos mostraram um perfil de baixa escolaridade, baixa renda e situação de dependência do benefício previdenciário (Tabelas 1, 2 e 3).

Tabela 1. Escolaridade

\begin{tabular}{lcc}
\hline Escolaridade & Frequência & Porcentagem \\
\hline Não alfabetizado & 53 & 35,3 \\
Ensino Fundamental Incompleto & 71 & 47,3 \\
Ensino Fundamental Completo & 5 & 3,3 \\
Ensino Médio Incompleto & 7 & 4,6 \\
Ensino Médio Completo & 12 & 8 \\
Ensino Superior Incompleto & 0 & 0 \\
Ensino Superior Completo & 2 & 1,3 \\
\hline Total & 150 & 100 \\
\hline
\end{tabular}

Tabela 2. Renda

\begin{tabular}{lcc}
\hline Renda & Frequência & Porcentagem \\
\hline Sem renda & 4 & 2,6 \\
Até meio salário mínimo & 7 & 4,6 \\
Entre meio e um salário mínimo & 65 & 43,3 \\
Entre um e dois salários mínimos & 52 & 34,6 \\
Entre três e cinco salários mínimos & 20 & 13,3 \\
Acima de cinco salários mínimos & 2 & 1,3 \\
\hline Total & 150 & 100 \\
\hline
\end{tabular}

Tabela 3. Situação funcional

\begin{tabular}{lcc}
\hline Situação funcional & Frequência & Porcentagem \\
\hline Empregado & 9 & 6 \\
Desempregado & 11 & 7,3 \\
Autônomo & 16 & 10,6 \\
Benefício previdenciário & 100 & 66,6 \\
Não se aplica & 14 & 9,3 \\
\hline Total & 150 & 100 \\
\hline
\end{tabular}

Os diagnósticos médicos que aparecem com maior frequência foram: doenças do aparelho circulatório (19\%), doenças do aparelho digestivo (16\%) e doenças do sistema nervoso (14\%) (Figuras 1 e 2).

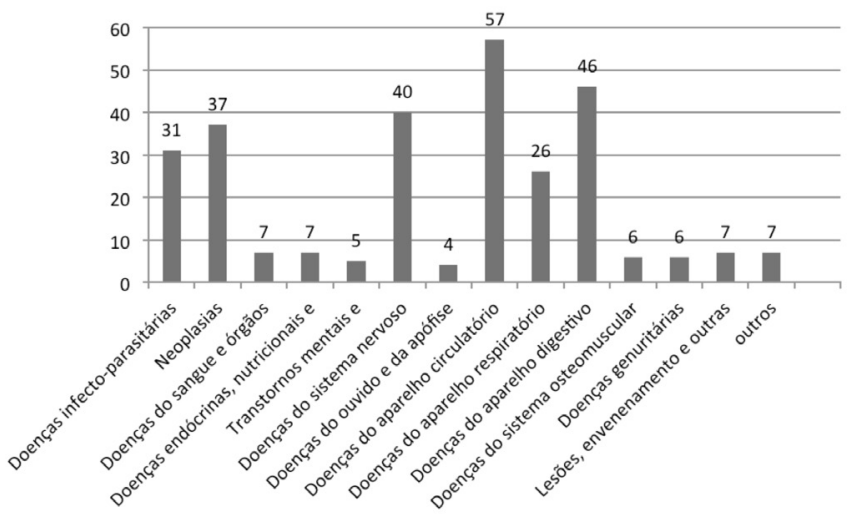

Figura 1. Diagnósticos médicos divididos em grupos, amostra em números absolutos (CID 10)

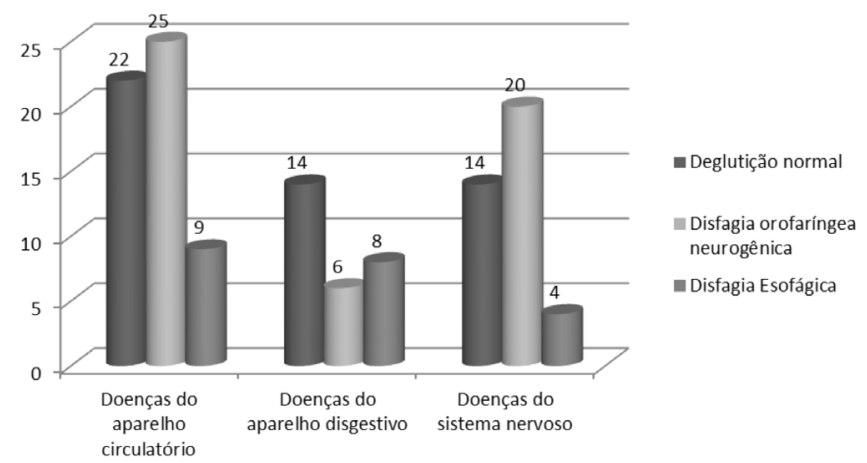

Figura 2. Relação entre diagnósticos médicos e diagnósticos fonoaudiológicos encontrados na amostra (resultado em números absolutos)

É importante observar que, dos 150 participantes, 97 $(64,7 \%)$ apresentaram dois diagnósticos médicos, 43 (28,7\%) apresentaram três diagnósticos, $8(5,3 \%)$ apresentaram quatro diagnósticos e $1(7 \%)$ apresentou cinco diagnósticos.

Com relação à forma de acesso ao paciente, dos 150 atendidos, 129 (86\%) foram por captação espontânea dos residentes de Fonoaudiologia, no momento da admissão dos pacientes no PS, 14 (9,3\%) foram via parecer médico e apenas $7(4,7 \%)$, via parecer de outro profissional da saúde (Figura 3).

Segundo o livro de registros do serviço de Fonoaudiologia, na amostra de 150 pacientes foram contabilizados 563 atendimentos realizados pelos residentes, sendo que, 205 destes, foram avaliações e 358 foram terapias. Esse resultado aponta para a média de 1,3 avaliações por paciente e 2,3 terapias por paciente.

Com relação aos diagnósticos fonoaudiológicos realizados durante esse período, os principais foram: deglutição normal/ funcional (44\%), disfagia orofaríngea neurogênica $(29,3 \%)$ e disfagia esofágica $(24,6 \%)$ (Figura 4). 


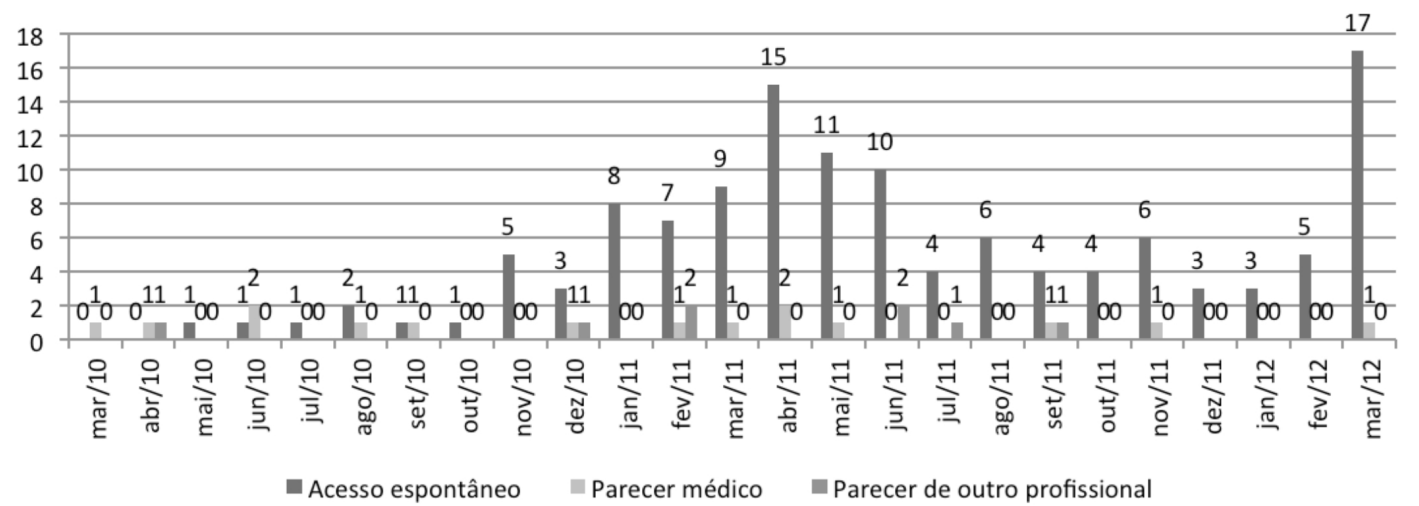

Figura 3. Atendimentos em números absolutos realizados pelos residentes de Fonoaudiologia no período da pesquisa (março/2010 a março/2012)

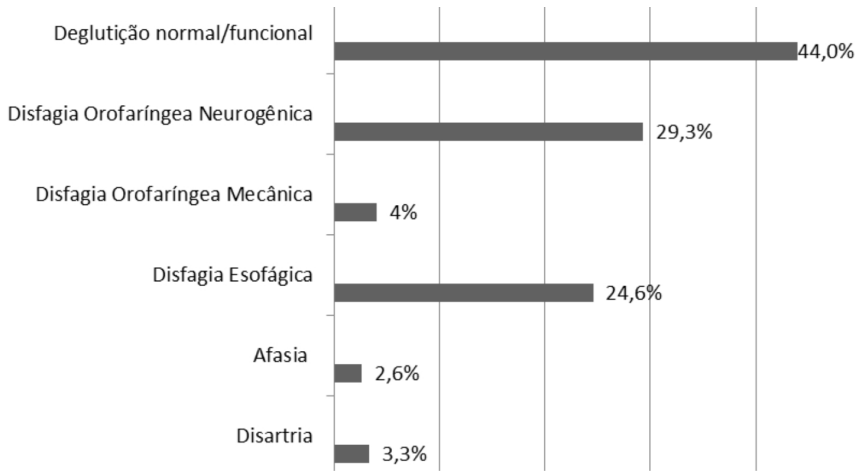

Figura 4. Diagnósticos fonoaudiológicos realizados pelas residentes

\section{DISCUSSÃO}

Os dados obtidos sobre a predominância de disfagia orofaríngea neurogênica nos grupos de doenças do aparelho circulatório e de disfagia esofágica no grupo de doenças do aparelho digestivo (Figura 2), concordam com a pesquisa realizada em 2009, em uma instituição hospitalar de Pernambuco, que aponta para alterações encontradas na deglutição de idosos, até mesmo na ausência de alterações neurológicas, em decorrência do envelhecimento dos órgãos fonoarticulatórios ${ }^{(23)}$. Essas alterações são relacionadas, principalmente, à fase orofaríngea da deglutição e são explanadas detalhadamente nas literaturas da área de motricidade orofacial ${ }^{(3,4)}$.

Os 20 pacientes com diagnóstico de doença do sistema nervoso que apresentaram disfagia orofaríngea neurogênica, confirmam estudos em pacientes com acometimento neurológico e apontam para um índice de $50 \%$ de prevalência desse tipo de disfagia, na ocorrência de episódios de Acidente Vascular Cerebral (AVC) $)^{(8,9)}$.

Quanto à predominância de disfagia esofágica e sua relação com as doenças do aparelho digestivo, a literatura mostra que esse tipo de alteração é frequente em várias doenças tais como o Megaesôfago Chagásico e o Divertículo de Zenker ${ }^{(24)}$.

A alta prevalência de disfagia identificada nesta amostra e em outros estudos, ressalta a importância da participação da Fonoaudiologia na estruturação de uma rede de atendimento ao paciente disfágico, desde a avaliação nas unidades de pronto atendimento até cuidados domiciliares, especialmente, quando se trata de idosos ${ }^{(16)}$.

De acordo com o Instituto Brasileiro de Geografia e Estatísticas (IBGE), no Brasil existem mais de 20 milhões de idosos, ou seja, aproximadamente $8,6 \%$ da população do país tem idade igual ou superior a 60 anos. Segundo o MS, as doenças do aparelho circulatório são as que mais atingem essa faixa etária e representam $37 \%$ das causas de mortes entre os idosos ${ }^{(25)}$. Esses dados foram confirmados por este estudo, uma vez que 56,7\% dos pacientes da amostra eram idosos, demonstrando, ainda, que o diagnóstico médico que aparece com maior frequência é o de doenças do aparelho circulatório (19\%), seguido pelo diagnóstico de doenças do aparelho digestivo $(16 \%)$ e do sistema nervoso $(14 \%)$.

O perfil socioeconômico dos pacientes deste estudo demonstrou que a maioria tem baixa escolaridade $(47,3 \%)$, baixa renda $(43,3 \%)$ e depende do benefício previdenciário $(66,6 \%)$, concordando com uma pesquisa realizada em 2011, em Fortaleza - Ceará, que apresenta a mesma similaridade no perfil dos idosos cadastrados na rede de unidade básica de saúde da cidade ${ }^{(26)}$.

Nos Estados Unidos, um estudo publicado em 2009 mostrou que, durante o período de 1996 a 2005, houve um aumento no número de usuários dos serviços de urgência e emergência, de $12,7 \%$ para $13,8 \%$ e relatou, ainda, que a maioria desses usuários pertence à terceira idade ${ }^{(27)}$. Nas bases de dados pesquisadas não foram encontrados mais trabalhos com características semelhantes, o que impossibilitou melhores comparações.

Um estudo realizado em 2011, mostra que, dos 20.390 fonoaudiólogos registrados no Cadastro Nacional de Estabelecimentos de Saúde (CNES), apenas 0,34\% atuam nesse tipo de serviço. Segundo os autores, de 2005 a 2011 houve aumento de fonoaudiólogos atuando no atendimento de urgência e emergência, principalmente após a criação dos programas de residência multiprofissional em saúde, mas o número de profissionais ainda não é suficiente para suprir a demanda $^{(28)}$.

Outro ponto de relevância neste trabalho, foi mostrar o aumento significativo do número de atendimentos fonoaudiológicos realizados no PS durante os períodos em que havia 
residentes de Fonoaudiologia fixos no local. Até o início do programa residência multiprofissional, os atendimentos eram realizados apenas sob pedidos de parecer médico (Figura 3).

A natureza retrospectiva do estudo limitou a capacidade de coletar informações adicionais, não sendo possível, também, realizar melhores comparações com dados de outros estudos, devido à escassez de publicações sobre o tema.

\section{CONCLUSÃO}

A faixa etária associada a doenças de base prevalentes pode levar à disfagia, o que agrava o quadro clínico e prolonga o período de permanência do sujeito na instituição, sendo de extrema importância a presença do fonoaudiólogo nos serviços de urgência e emergência para avaliar, diagnosticar e tratar os distúrbios da deglutição.

Este estudo mostra que, mais de $50 \%$ da população atendida no PS do HC/UFG durante o período pesquisado, é disfágica ou apresenta riscos para o desenvolvimento desse distúrbio, levantando a hipótese de que o mesmo fato ocorra em todo o país.

Tendo como base o exposto, a equipe multiprofissional pode iniciar e desenvolver planos de assistência focados nessas circunstâncias e discutir, posteriormente, sua continuidade ou encaminhamento para outras instituições de saúde.

\section{REFERÊNCIAS}

1. Lessa F, Ferrite MTPCS.Fonoaudiologia e Epidemiologia. In Fernandes FDM, Mendes BCA, Navas ALPGP (Org.). Tratado de Fonoaudiologia. 2a. ed. São Paulo: Roca; 2010. p. 34-44.

2. Brasil. Diário Oficial Imprensa Nacional $n^{\circ} .239$ - DOU de 09/12/08, p. 163. Conselho Federal de Fonoaudiologia. Resolução $n^{\circ} 356$, de 6 de dezembro de 2008. [Internet] [citado 2012 Nov. 20]. Disponível em: ftp://ftp.saude.sp.gov.br/ftpsessp/bibliote/informe_ eletronico/2008/iels.dez.08/iels234/U_RS-CFFa-356_061208.pdf

3. Rofes L, Arreola V, Almirall J, Cabré M, Campins L, García-Peris P, et al. Diagnosis and management of oropharyngeal dysphagia and Its nutritional and respiratory complications in the elderly. Gastroenterol Res Pract. 2011;2011:1-14.

4. Prodomo LPV, Angelis EC, Barros ANP. Avaliação clínica fonoaudiológica das disfagias. In: Jotz GP, Angelis EC, Barros APB. Tratado da deglutição e disfagia: no adulto e na criança. Rio de Janeiro: Revinter; 2010. p. 61-7.

5. Souza BBA, Martins C, Campos DJ, Balsini ID, Meyer LR. Nutrição e disfagia: guia para profissionais. Curitiba: Nutroclínica; 2003. p. 17-22.

6. Portal da Saúde. Sistema Único de Saúde. [Internet] [citado 2012 Nov. 20]. Disponível em: http://portal.saude.gov.br/portal/saude/ profissional/visualizar_texto.cfm?idtxt=31877

7. Silvério CC, Hernandez AM, Gonçalves MIR. Ingesta oral do paciente hospitalizado com disfagia orofaríngea neurogênica. Rev CEFAC. 2010;12(6):964-70.

8. Moraes DP, Andrade CRF. Indicadores de qualidade para o gerenciamento da disfagia em Unidades de Internação Hospitalar. J Soc Bras Fonoaudiol. 2011;23(1):89-94.

9. Lawrence ES, Coshall C, Dundas R, Stewart J, Rudd AG, Howard $\mathrm{R}$, et al. Estimates of the prevalence of acute stroke impairments and disability in a multiethnic population. Stroke. 2001;32(6):1279-84.

10. Kröger E, Tourigny A, Morin D, Côté L, Kergoat MJ, Lebel P, et al. Selecting process quality indicators for the integrated care of vulnerable older adults affected by cognitive impairment or dementia. BMC Health Serv Res. 2007;7:195.

11. Martino R, Foley N, Bhogal S, Diamant N, Speechley M, Teasell R. Dysphagia after stroke: incidence, diagnostic and pulmonary complications. Stroke. 2005;36(12):2756-63.

12. Geeganage C, Beavan J, Ellender S, Bath PM. Interventions for dysphagia and nutritional support in acute and subacute stroke. Cochrane Database Syst Rev. 2012;10:13p.

13. Baroni AF, Fabio SR, Dantas RO. Risk factors for sawallowing dysfunction in stroke patients. Arq Gastroentrol. 2012;49(2):118-24.

14. Ashley J, Duggan M, Suticliffe N. Speech, language, and swallowing disorders in the older adult. Clin Geriatr Med. 2006;22(2):291-310.

15. Abdulmassih EMS, Macedo Filho ED, Santos RS, Jurkiewicz AL. Evolução de pacientes com disfagia orofaríngea em ambiente hospitalar. Arq Int Otorrinolaringol. 2009;13(1):55-62.

16. Farneti D, Consolmagno P. The Swallowing Centre: rationale for a multidisciplinary management. Acta Otorhinolaryngol Ital. 2007;27(4):200-7.

17. Saar SRC, Trevizan MA. Papéis profissionais de uma equipe de saúde: visão de seus integrantes. Rev Latino-Am Enfermagem. 2007;1(15):106-12.

18. Santoro PP, Furia CLB, Forte AP, Lemos EM, Garcia RI, Tavares RA, et al. Otolaryngology and speech therapy evaluation in the assessment of oropharyngeal dysphagia: a combined protocol proposal. Braz J Otorhinolaryngol. 2011;77(2):201-13.

19. Marques CHD, André C, Rosso ALZ. Disfagia no AVE agudo: revisão sistemática sobre métodos de avaliação. Acta Fisiátrica. 2008;15(2):106-10.

20. Itaquy RB, Favero SR, Ribeiro MC, Barea LM, Almeida ST, Mancopes R. Disfagia e acidente vascular cerebral: relação entre o grau de severidade e o nível de comprometimento neurológico. J Soc Bras Fonoaudiol. 2011;23(4):385-9.

21. Brasil. Ministério da Saúde. Portaria n ${ }^{\circ} 3.318$, de 28 de outubro de 2010. [Internet] [citado 2012 Nov. 20]. Disponível em: http://bvsms. saude.gov.br/bvs/saudelegis/gm/2010/prt3318_28_10_2010.html.

22. Organização Mundial da Saúde. CID-10 Classificação Estatística Internacional de Doenças e Problemas Relacionados à Saúde. 10a . rev. São Paulo: Universidade de São Paulo; 1997. vol.1.

23. Lima RMF, Amaral AKFJ, Aroucha EBL, Vasconcelos TMJ, Silva HJ, Cunha DA. Adaptações na mastigação, deglutição e fonoarticulação em idosos de instituição de longa permanência. Rev CEFAC. 2009;11(3):405-22.

24. Nascimento WV, Cassiani RA, Dantas RO. Disfagia em pacientes com doença de Chagas e divertículo de Zenker. Rev Soc Bras Fonoaudiol. 2010;15(2):277-81.

25. Portal Brasil. Sistema Único de Saúde. País investe em prevenção 
a doenças da terceira idade. [citado 2012 Nov. 20] Disponível em: http://www.brasil.gov.br/saude/2012/04/pais-investe-em-prevencaoa-doencas-da-terceira-idade.

26. Clares JWB, Freitas MC, Almeida PC, Galiza FT, Queiroz TA. Perfil de idosos cadastrados numa Unidade Básica de Saúde da Família de Fortaleza-CE. Rev Rene. 2011;12(n.esp.):988-94.
27. Xu TK, Nelson BK, Berk S. The changing profile of patients who used emergency department services in the United States: 1996 to 2005. Ann Emerg Med. 2009;54(6):805-10.

28. Costa KN, Guimarães VC. Fonoaudiologia nos serviços de urgência e emergência do Brasil: série histórica de 2005 a 2011. Distúrb Comum. 2012;24(1):69-75.

Anexo 1. Perfil do Paciente Atendido pela Fonoaudiologia na Unidade de Urgência e Emergência de um Hospital Universitário

Formulário $n^{\circ}$ Data:

Dados sociodemográficos

Número do prontuário:

Faixa etária:

1. ( ) Adulto

2. ( ) Idoso

Sexo:

1. ( ) Feminino

2. ( ) Masculino

Procedência

1. ( ) Goiânia e região metropolitana

2. ( ) Interior de Goiás

3. ( ) Outro estado

Renda

1. ( ) Sem renda

2. ( ) Até meio salário mínimo

3. ( ) Entre meio e um salário mínimo

4. ( ) Entre um e dois salários mínimos

5. ( ) Entre três e cinco salários mínimos

6. ( ) Acima de cinco salários mínimos

\section{Escolaridade}

1. ( ) Não alfabetizado

2. ( ) Ensino fundamental incompleto

3. ( ) Ensino fundamental completo

4. ( ) Ensino médio incompleto

5. ( ) Ensino médio completo

6. ( ) Ensino superior incompleto

7. ( ) Ensino superior completo
Doenças encontradas

1. ( ) Doenças Infecto parasitárias

2. ( ) Neoplasias

3. ( ) Doenças do sangue e órgãos hematopoiéticos

4. ( ) Doenças endócrinas, nutricionais e metabólicas

5. ( ) Transtornos mentais e comportamentais

6. ( ) Doenças do sistema nervoso

7. ( ) Doenças do ouvido e apófise mastoidea

8. ( ) Doenças do aparelho circulatório

9. ( ) Doenças do aparelho respiratório

10. ( ) Doenças do aparelho digestivo

11. ( ) Doenças do sistema osteomuscular e tecido conjuntivo

12. ( ) Doenças geniturinárias

13. ( ) Lesões, envenenamento e outras causas externas

14. ( ) Outros

Acesso ao paciente

1. ( ) Espontâneo

2. ( ) Via parecer médico

3. ( ) Via parecer de outro profissional

Diagnóstico Fonoaudiológico

1. ( ) Deglutição normal/funcional

2. ( ) Disfagia orofaríngea neurogênica

3. ( ) Disfagia orofaríngea mecânica

4. ( ) Disfagia esofágica

5. ( ) Afasia

6. ( ) Disartria 